\title{
Conteúdo de carbono e atributos químicos de Latossolo sob cana-de-açúcar colhida com e sem queima
}

\author{
Benedito Luiz Correia(1) e Luis Reynaldo Ferracciú Alleoni(2) \\ (1)Universidade Federal Rural de Pernambuco, Colégio Agrícola Dom Agostinho Ikas, Avenida Dr. Francisco Corrêa, no 643, \\ CEP 54735-000 São Lourenço da Mata, PE. E-mail: bcorreia@codai.ufrpe.br (2)Universidade de São Paulo, Escola Superior de Agricultura \\ Luiz de Queiroz, Avenida Pádua Dias, no 11, CEP 13418-900 Piracicaba, SP. E-mail: alleoni@esalq.usp.br
}

Resumo - O objetivo deste trabalho foi avaliar o acúmulo de carbono e os atributos químicos de um Latossolo Vermelho distrófico em sistemas de colheita da cana-de-açúcar com (CQ) e sem queima (SQ), em experimento de longa duração. Em 2009, após 14 anos da implantação do experimento em Pradópolis, SP, amostras de solo foram coletadas na linha de plantio, a $0,25 \mathrm{~m}$ da linha e na entrelinha, nas profundidades de $0-0,05,0,05-0,1$, $0,1-0,2,0,2-0,3$ e $0,3-0,4 \mathrm{~m}$. Foram determinados os teores de carbono orgânico total (COT), $\mathrm{Ca}^{2+}, \mathrm{Mg}^{2+} \mathrm{e} \mathrm{K}^{+}$ trocáveis, P-resina e P-Mehlich-1. Em geral, no sistema SQ, os teores de COT foram superiores na linha e a $0,25 \mathrm{~m}$ da linha, até a profundidade de $0,3 \mathrm{~m}$. Na entrelinha, o acúmulo de carbono orgânico limitou-se às duas primeiras camadas superficiais. O sistema SQ também favoreceu a capacidade de troca catiônica e os valores de $\mathrm{Ca}^{2+}, \mathrm{Mg}^{2+}$ e saturação por bases, independentemente de locais de amostragem, nas duas primeiras camadas superficiais. O teor de $\mathrm{P}$ disponível foi pouco afetado pelos sistemas de colheita, com valores maiores no CQ. A colheita sem queima favorece o acúmulo de carbono orgânico e o aumento da fertilidade do solo, exceto quanto aos teores de P disponível.

Termos para indexação: cana-soca, capacidade de troca catiônica, carbono orgânico, fertilidade do solo, matéria orgânica, palha.

\section{Carbon content and chemical attributes of Oxisol under sugarcane harvested with and without burning}

\begin{abstract}
The objective of this work was to evaluate the chemical attributes of a Rhodic Hapludox according to the sugarcane harvest system, with (CQ) and without burning (SQ), in long-term experiment. In 2009, after 14 years of establishment of a field experiment in Pradópolis, SP, Brazil, soil samples were taken at the planting row, at $0.25 \mathrm{~m}$ from the row, and between rows, at $0-0.05,0.05-0.1,0.1-0.2,0.2-0.3$, and $0.3-0.4-\mathrm{m}$ depth. Total organic carbon content (TOC), exchangeable $\mathrm{Ca}^{2+}, \mathrm{Mg}^{2+}$ and $\mathrm{K}^{+}$, and resin and Mehlich-1 available $\mathrm{P}$ were determined. In general, the SQ system had higher levels of TOC in the row, and at 0.25 from the row, to a depth of $0.3 \mathrm{~m}$. Between lines, the accumulation of carbon was restricted to the first two layers of the soil. SQ system also favored cation exchange capacity and the values of $\mathrm{Ca}^{2+}, \mathrm{Mg}^{2+}$, and base saturation, regardless of sampling location, at the first two layers. Available contents of $\mathrm{P}$ were little affected by harvesting systems, with higher values found in the CQ system. Harvesting without burning increases carbon accumulation and soil fertility, except for available $P$.
\end{abstract}

Index terms: ratoon cane, cation exchange capacity, organic carbon, soil fertility, organic matter, straw.

\section{Introdução}

No sistema de colheita de cana-de-açúcar sem queima, são depositados, sobre o solo, de 10 a $15 \mathrm{Mg} \mathrm{ha}^{-1}$ por ano de palha, cuja decomposição afeta o ciclo do carbono e a dinâmica da matéria orgânica (MO) (Luca et al., 2008). O aporte de MO é de fundamental importância em solos tropicais de regiões úmidas, na sua maioria altamente intemperizados e ácidos, pois melhora a disponibilidade de nutrientes para as culturas, a capacidade de troca de cátions
(CTC) e a complexação de elementos tóxicos (Bayer \& Mielniczuk, 2008).

A preservação e a intensidade dos efeitos benéficos da MO na fertilidade estão associados à formação de complexos argilo-orgânicos e têm correlação positiva com o teor de argila do solo (Zinn et al., 2005). A disponibilização dos nutrientes imobilizados na camada de palha diretamente em contato com o solo depende da composição química do material, do tempo de decomposição e de fatores ambientais, como temperatura, disponibilidade hídrica e oxigênio

Pesq. agropec. bras., Brasília, v.46, n.8, p.944-952, ago. 2011 
(Silva \& Mendonça, 2007). Sabe-se que, nas áreas submetidas à esse manejo de colheita, ocorre elevação da CTC e da biodisponibilidade de nutrientes (Oliveira et al., 1999; Canellas et al., 2010).

Em virtude do tráfego das colhedoras de cana-de-açúcar, que intensifica a compactação nas entrelinhas de plantio (Wood, 1991), e da limitação à aplicação de adubos orgânicos e minerais na superfície do solo por causa da palha, $75 \%$ das raízes da cana-de-açúcar ficam concentradas nos $40 \mathrm{~cm}$ superficiais, com uma densa distribuição lateral até $20 \mathrm{~cm}$ da linha de plantio (Alvarez et al., 2000). No entanto, poucos estudos, que levem em consideração os efeitos da palha na fertilidade do solo, são conduzidos para reavaliar a localização e as profundidades para amostragem do solo, com vistas a recomendações de adubação mais econômicas e eficazes.

O primeiro passo na avaliação da fertilidade do solo é a amostragem adequada, especialmente em culturas semiperenes, como a cana-de-açúcar, na qual há uma faixa próxima à linha bem diferenciada de outra faixa, na entrelinha. Raij et al. (1997), recomendam a amostragem nas entrelinhas da soqueira, enquanto Vitti \& Mazza (2002) recomendam amostragem a 20-25 cm da linha de plantio. Segundo os autores, pode ocorrer superestimativa dos teores de $\mathrm{Ca}^{2+}, \mathrm{Mg}^{2+}$ e da soma e saturação por bases, em amostras coletadas na entrelinha, e superestimativa dos teores de $\mathrm{PeK}^{+}$em amostras tiradas da linha de plantio. De acordo com Malavolta (1992), $30 \%$ das subamostras da área devem ser coletadas na linha e 70\% na entrelinha. Raij et al. (1997) e Vitti \& Mazza (2002) recomendam a amostragem na camada de 0-0,2 m, em colheita com queima e corte manual.

As pesquisas com outras culturas no sistema de plantio direto têm avançado, e diversos pesquisadores reconhecem as alterações de fertilidade promovidas nas camadas mais superficiais do solo $(0,2 \mathrm{~m})$. A Comissão de Fertilidade de Solo do Núcleo Regional Sul da Sociedade Brasileira de Ciência do Solo recomenda, desde 1997, a amostragem na camada de 0-0,1 m em áreas sob plantio direto por mais de cinco anos (Manual de adubação e de calagem para os Estados do Rio Grande do Sul e de Santa Catarina, 2004).

O objetivo desse trabalho foi avaliar o acúmulo de carbono e os atributos químicos de um Latossolo Vermelho distrófico em sistemas de colheita da canade-açúcar com e sem queima, em experimento de longa duração.

\section{Material e Métodos}

As amostras de solo foram coletadas em Pradópolis, SP. O solo da região é classificado como Latossolo Vermelho distrófico muito argiloso (Santos et al., 2006), com teor médio de $656 \mathrm{~g} \mathrm{~kg}^{-1}$ de argila, até $40 \mathrm{~cm}$ de profundidade. A fração argila é constituída principalmente de caulinita, gibbsita, goethita e hematita, nas quantidades de 294, 196, 70 e $59 \mathrm{~g} \mathrm{~kg}^{-1}$, respectivamente. A caracterização química do solo, antes da instalação do experimento, encontra-se na Tabela 1. O experimento foi instalado em 1995, na Fazenda Aparecida, da Usina São Martinho (21 ${ }^{\circ} 23^{\prime} \mathrm{S}$; $47^{\circ} 59^{\prime} \mathrm{W}$; a $620,3 \mathrm{~m}$ de altitude), em gleba cultivada com cana-de-açúcar desde 1950, após retirada da vegetação nativa. A área recebeu $2 \mathrm{Mg} \mathrm{ha}^{-1}$ de calcário e adubação de plantio com $500 \mathrm{~kg} \mathrm{ha}^{-1}$ da fórmula NPK 10-25-25. O primeiro corte foi realizado em 1996, com a palha tendo sido depositada em área total, nas parcelas colhidas sem queima. O mesmo manejo da palha foi mantido em todos os cortes subsequentes. A adubação de soqueira, de 1996 a 2003, foi feita com $600 \mathrm{~kg} \mathrm{ha}^{-1}$ da fórmula 15-05-25. Na renovação do canavial, em 2003, a área recebeu $2 \mathrm{Mg} \mathrm{ha}^{-1}$ de calcário. O manejo da fertilidade do solo consistiu apenas de adubação mineral de plantio $\left(26,56\right.$ e $84 \mathrm{~kg} \mathrm{ha}^{-1}$ de $\mathrm{N}, \mathrm{P}_{2} \mathrm{O}_{5}$ e $\mathrm{K}_{2} \mathrm{O}$, respectivamente) e de soqueira, no período de 2004-2008 (em média 105 e $93 \mathrm{~kg} \mathrm{ha}^{-1} \mathrm{de}$ $\mathrm{Ne} \mathrm{K}_{2} \mathrm{O}$, respectivamente).

Utilizou-se o delineamento experimental de blocos ao acaso, em parcelas subdivididas, com seis repetições. As parcelas foram constituídas por cana-de-açúcar colhida mecanicamente, sem queima (SQ), e colhida manualmente, com queima (CQ). As subparcelas foram constituídas pelos locais de amostragem: na linha de plantio, a $0,25 \mathrm{~m}$ da linha e na entrelinha. As parcelas foram compostas de 22 linhas de plantio com $50 \mathrm{~m}$ de comprimento, e área útil de dez linhas com $30 \mathrm{~m}$ de comprimento. Em cada local de amostragem, foram avaliadas as camadas: $0-0,05,0,05-0,1,0,1-0,2$, $0,2-0,3$ e $0,3-0,4 \mathrm{~m}$.

As amostras do solo foram coletadas em janeiro de 2009, três meses após o quinto corte da soqueira. Em cada parcela, foram coletadas 20 amostras simples que deram origem a uma amostra composta para cada posição e profundidade.

As amostras compostas foram secas ao ar e peneiradas em malha de $2 \mathrm{~mm}$, para obtenção de terra fina seca ao ar (TFSA). O teor de $\mathrm{C}$ total foi 
determinado por combustão a seco em autoanalisador elementar Flash EA 1112 (Thermo, Rodano, Milão, Itália). Também foram determinados: $\mathrm{pH}$ em $\mathrm{CaCl}_{2}$ a $0,01 \mathrm{~mol} \mathrm{~L} \mathrm{~L}^{-1}$, na proporção TFSA:solução de 1:2,5; $\mathrm{K}^{+}$trocável extraído por Mehlich-1 $(\mathrm{HCl} \mathrm{0,05+}$ $\mathrm{H}_{2} \mathrm{SO}_{4} 0,0125 \mathrm{~mol} \mathrm{~L}^{-1}$ ), determinado por fotometria de chama; $\mathrm{Ca}^{2+}$ e $\mathrm{Mg}^{2+}$ extraídos por resina trocadora de íons, determinados por espectrofotometria de absorção atômica; P-disponível extraído pela resina trocadora de íons (P-resina), quantificado por colorimetria (Raij et al., 2001); e P-disponível extraído por Mehlich-1 (P Mehlich), também quantificado por colorimetria. Foram calculadas soma e saturação por bases (V), acidez total $(\mathrm{H}+\mathrm{Al})$ extraída com acetato de amônio a $\mathrm{pH} 7,0$, e a CTC total a $\mathrm{pH} 7,0$.

As análises estatísticas foram realizadas pelo procedimento GLM do SAS, versão 9.2 (SAS Institute, 1997), e as médias foram comparadas pelo teste de Tukey, a 5\% de probabilidade. Para que se obtivesse homogeneidade de variância e normalidade dos erros, os dados foram transformados da seguinte maneira: 1/COT; $\log \mathrm{pH} \mathrm{CaCl}_{2} ;\left(\mathrm{Al}^{3+}\right)^{0,5} ;(\mathrm{m})^{0,5} ; \log \mathrm{H}+\mathrm{Al}$; $1 /(\mathrm{P}-\text { resina })^{0,5} ; 1 /(\mathrm{P} \text { Mehlich })^{0,5} ; 1 /\left(\mathrm{Ca}^{2+}\right)^{0,5} ; \log \mathrm{Mg}^{2+}$; $\mathrm{K}^{+(0,05)} ; \mathrm{CTC}^{-2} ; \log \mathrm{V}$.

\section{Resultados e Discussão}

O teor de carbono orgânico total (COT) na área SQ foi superior ao da CQ nas profundidades de $0-0,05$ e $0,05-0,1 \mathrm{~m}$, em todas as posições de amostragem (Figura 1). Na média das três posições, o teor de COT nas amostras de solo coletadas no sistema SQ foi $28 \%$ maior do que no CQ, na camada de 0-0,05 m (SQ $\left.=29 \mathrm{~g} \mathrm{~kg}^{-1} ; \mathrm{CQ}=22,7 \mathrm{~g} \mathrm{~kg}^{-1}\right)$, enquanto na camada subsequente $(0,05-0,1 \mathrm{~m})$ a diferença foi de $22 \%\left(\mathrm{SQ}=25,3 \mathrm{~g} \mathrm{~kg}^{-1} ; \mathrm{CQ}=20,8 \mathrm{~g} \mathrm{~kg}^{-1}\right)$. Razafimbelo et al. (2006), ao avaliar a mesma área experimental após seis anos de cultivo, observaram que o teor de COT foi $20 \%$ maior no solo no sistema SQ do que no CQ, na camada de $0-0,05 \mathrm{~m}$, e $9 \%$ maior na camada de 0,05-0,1 m. Esses resultados são condizentes com os obtidos no presente trabalho, pois houve aumento no acúmulo de carbono orgânico de 6 para 14 anos de manejo sem queima da palha da cana-de-açúcar.

Em geral, no sistema SQ, os teores de COT foram superiores na linha e a $0,25 \mathrm{~m}$, até a profundidade de 0,3 m (Figura 1). Na entrelinha, o acúmulo de carbono orgânico limitou-se às duas primeiras camadas superficiais. Como a palha foi distribuída em área total, esses resultados provavelmente estão associados à maior densidade de raízes no tratamento SQ (Anghinoni, 2007). Na entrelinha, a estabilização do COT pela formação de complexos organominerais (Zinn et al., 2005) pode explicar o maior teor nas camadas superficiais, no tratamento SQ. Na profundidade de $0,3-0,4 \mathrm{~m}$, não houve diferença nos valores de COT entre os sistemas, em todas as posições. Canellas et al. (2010), ao avaliar canavial cultivado sem queima da palha há mais de 50 anos, em um Cambissolo com $270 \mathrm{~g} \mathrm{~kg}^{-1}$ de argila, relataram elevação nas quantidades de COT em até $0,4 \mathrm{~m}$ de profundidade. Nessas condições, a percolação de compostos orgânicos solúveis é favorecida.

Observou-se o seguinte gradiente de COT, no sistema SQ: linha $=0,25 \mathrm{~m}>$ entrelinha, na camada de $0-0,05 \mathrm{~m}$. Em geral, nas demais profundidades, foram verificados maiores teores de COT na linha do que nas demais posições (Tabela 2). Além da adição pela distribuição da palha, o acúmulo de MO na linha de plantio decorreu da adição de maior quantidade de raízes, da liberação de exsudatos radiculares e de lavagens de constituintes solúveis da planta pelas chuvas (Silva \& Mendonça, 2007).

No sistema SQ, os valores de CTC foram maiores do que os observados no sistema CQ, independentemente das posições e profundidades de amostragem, com efeito mais pronunciado na camada de 0-0,05 m (Figura 1). Czycza (2009) e Canellas et al. (2010) obtiveram resultados semelhantes em Cambissolo e Latossolo, respectivamente, cultivados com cana-de-açúcar. No entanto, Mendonza et al. (2000) não observaram diferenças na CTC de Argissolo arenoso com baixo

Tabela 1. Análises químicas do solo após o primeiro corte da cana-de-açúcar, em 1996.

\begin{tabular}{|c|c|c|c|c|c|c|c|c|c|c|c|c|}
\hline $\begin{array}{c}\text { Camadas } \\
(\mathrm{m})\end{array}$ & $\begin{array}{c}\mathrm{pH} \\
\text { em } \mathrm{CaCl}_{2}^{(1)}\end{array}$ & $\begin{array}{c}\mathrm{MO} \\
\left(\mathrm{g} \mathrm{dm}^{-3}\right)\end{array}$ & $\begin{array}{c}\mathrm{P}^{(2)} \\
\left(\mathrm{mg} \mathrm{dm}^{-3}\right)\end{array}$ & $\mathrm{K}^{+}$ & $\mathrm{Ca}^{2+}$ & $\mathrm{Mg}^{2+}$ & $\begin{array}{l}\mathrm{Al}^{3+} \\
\mathrm{mol}_{\mathrm{c}} \mathrm{C}\end{array}$ & $\mathrm{H}+\mathrm{Al}$ & SB & CTC & ----- (\%) ----- & $m$ \\
\hline $0-0,2$ & 4,7 & 37 & 13 & 2,1 & 22,8 & 6,7 & 2,0 & 47 & 32 & 79 & 40 & 6 \\
\hline $0,2-0,4$ & 4,9 & 33 & 19 & 1,7 & 26,1 & 7,5 & 1,3 & 42 & 35 & 77 & 46 & 3 \\
\hline
\end{tabular}

${ }^{(1)} 0,01$ mol L ${ }^{-1}$. ${ }^{(2)}$ P extraído por resina; SB, soma de bases; CTC, capacidade de troca de cátions; V, saturação por bases; m, saturação por alumínio. 
teor de MO, em função do manejo da cana-de-açúcar com ou sem queima, após seis anos de cultivo. Esses resultados evidenciam a importância da textura do solo no acúmulo de MO.

Em geral, os valores de CTC não foram influenciados pelos locais de amostragem, na maioria das camadas de solo amostradas, nos dois tipos de colheita (Figura 1). As exceções foram as camadas de $0-0,05$ $\mathrm{m}$, em ambos os sistemas de colheita, e a camada mais profunda $(0,3-0,4 \mathrm{~m})$ do solo sob sistema CQ. Nessas profundidades, verificaram-se maiores valores da CTC na linha de plantio, seguidos dos observados a $0,25 \mathrm{~m}$ da linha, e menores valores na entrelinha (Tabela 3). Em ambos os sistemas, os valores de CTC foram mais altos nas camadas superficiais, tendo diminuído nas camadas mais profundas. Os maiores valores de CTC nas camadas superficiais observados mesmo no sistema CQ podem ser explicados pela adição de palha parcialmente calcinada (Ball-Coelho et al., 1993).

Os teores de $\mathrm{Ca}^{2+}$ e de $\mathrm{Mg}^{2+}$ no sistema de colheita SQ superaram os observados em CQ, nas camadas de $0-0,05$ e $0,05-0,1 \mathrm{~m}$, em todos os locais de amostragem
(Figura 2). Como as mesmas quantidades de adubo e calcário foram aplicadas nos dois tratamentos, os maiores valores encontrados sob cana-de-açúcar SQ evidenciam a maior capacidade de retenção desses elementos no solo nesse sistema, o que está de acordo com os resultados de CTC e de acúmulo de COT observados. De 39 a $44 \%$ do total desses elementos imobilizado na palha pode ser liberado após um ano de

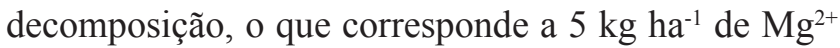
e $11 \mathrm{~kg} \mathrm{ha}^{-1}$ de $\mathrm{Ca}^{2+}$ por ano (Oliveira et al., 1999). Maiores concentrações de $\mathrm{Ca}^{2+}$ no manejo SQ também foram observadas nas camadas de $0,1-0,2,0,2-0,3$ e $0,3-0,4 \mathrm{~m}$. Não houve diferença entre os teores de $\mathrm{Mg}^{2+}$ nas profundidades de $0,1-0,2$ e $0,2-0,3 \mathrm{~m}$.

Possivelmente em razão do $\mathrm{K}^{+}$ser um nutriente rapidamente liberado no processo de decomposição da palha das culturas (Colonego et al., 2005), não foram observadas diferenças entre os sistemas SQ e CQ (Figura 2 ). No tratamento SQ, o elemento é adicionado ao solo pela ação das águas das chuvas (Rosolem et al., 2007) ou pela mineralização da palha (Oliveira et al., 1999), enquanto no sistema CQ, a adição de $\mathrm{K}^{+}$ocorre
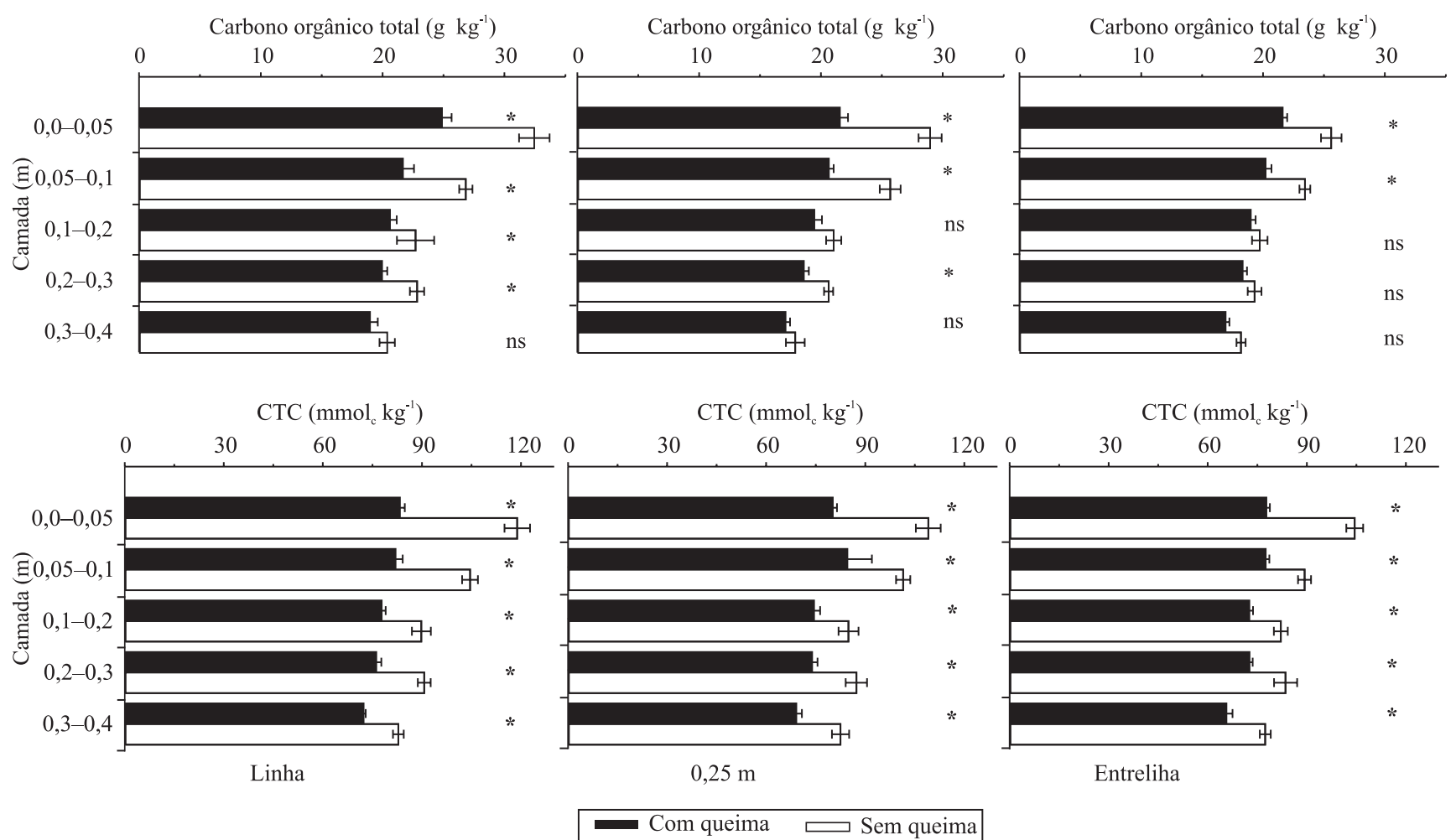

Figura 1. Valores de carbono orgânico total e capacidade de troca de cátions (CTC) em Latossolo Vermelho distrófico sob colheita de cana-de-açúcar com e sem queima. ns Não significativo. *Diferenças entre os sistemas de colheita com e sem queima pelo teste de Tukey a 5\% de probabilidade. As barras representam o erro-padrão da média. 
por meio das cinzas da queima da palha. Mendonza et al. (2000) também não encontraram diferença entre os teores de $\mathrm{K}^{+}$na cana-de-açúcar colhida nos sistemas com e sem queima, na camada de 0-0,1 m de um solo de tabuleiro costeiro, após cinco anos de cultivo. Nas camadas de $0,1-0,2$ e de $0,2-0,3 \mathrm{~m}$, os autores relataram alta concentração de $\mathrm{K}^{+}$no tratamento $\mathrm{CQ}$, e atribuíram o resultado à facilidade de transporte do elemento no solo em condições de baixa CTC.

Os maiores valores de $\mathrm{V}$ nas amostras de solo coletadas no sistema SQ, nas duas primeiras camadas superficiais, em todas as posições de amostragem (Figura 3), estão de acordo com os maiores teores de $\mathrm{Ca}^{2+}$ e de $\mathrm{Mg}^{2+}$ (Figura 2), nas mesmas posições e camadas. Em geral, nas camadas mais profundas, não houve diferença entre os dois sistemas de manejo. Mendonza et al. (2000) e Czycza (2009) não observaram diferenças em $\mathrm{V}$ de amostras de solo sob sistemas de cana-de-açúcar crua e queimada, independentemente das profundidades avaliadas. Os autores utilizaram faixas de profundidade mais largas na camada superficial $(0-0,1$ e $0-0,2 \mathrm{~m})$, o que provavelmente

Tabela 2. Carbono orgânico total e pH em um Latossolo Vermelho distrófico sob colheita de cana-de-açúcar com (CQ) e sem queima $(\mathrm{SQ})^{(1)}$.

\begin{tabular}{|c|c|c|c|c|c|c|c|}
\hline \multirow{2}{*}{$\begin{array}{l}\text { Camadas } \\
(\mathrm{m})\end{array}$} & \multicolumn{3}{|c|}{ Com queima } & \multicolumn{3}{|c|}{ Sem queima } & \multirow{2}{*}{$\begin{array}{l}\mathrm{CV} \\
(\%)\end{array}$} \\
\hline & Linha & $0,25 \mathrm{~m}$ & Entrelinha & Linha & $0,25 \mathrm{~m}$ & Entrelinha & \\
\hline \multicolumn{8}{|c|}{ Carbono orgânico total $\left(\mathrm{g} \mathrm{kg}^{-1}\right)$} \\
\hline $0,0-0,05$ & $24,9 \mathrm{aA}$ & $21,5 \mathrm{aB}$ & $21,6 \mathrm{aB}$ & $32,4 \mathrm{aA}$ & $29,0 \mathrm{aA}$ & $25,6 \mathrm{aB}$ & 7 \\
\hline $0,05-0,1$ & $21,7 \mathrm{bA}$ & $20,6 \mathrm{abA}$ & $20,2 \mathrm{abA}$ & $26,8 \mathrm{bA}$ & $25,7 \mathrm{bAB}$ & $23,4 \mathrm{aB}$ & 6 \\
\hline $0,1-0,2$ & $20,6 \mathrm{bcA}$ & $19,5 \mathrm{bcA}$ & $19,0 \mathrm{bcA}$ & $22,7 \mathrm{cA}$ & $21,0 \mathrm{cAB}$ & $19,7 \mathrm{bB}$ & 5 \\
\hline $0,2-0,3$ & $20,0 \mathrm{cbA}$ & $18,6 \mathrm{cB}$ & $18,3 \mathrm{cB}$ & $22,8 \mathrm{cA}$ & $20,6 \mathrm{cB}$ & $19,3 \mathrm{bC}$ & 4 \\
\hline$\underline{0,3-0,4}$ & $19,0 \mathrm{dA}$ & $17,1 \mathrm{dA}$ & $16,9 \mathrm{dA}$ & $20,4 \mathrm{dA}$ & $17,9 \mathrm{~dB}$ & $18,2 \mathrm{bB}$ & 6 \\
\hline $\mathrm{CV}(\%)$ & 4 & 4 & 5 & 5 & 5 & 6 & \\
\hline \multicolumn{8}{|c|}{$\mathrm{pH}$ em $\mathrm{CaCl}_{2} 0,01 \mathrm{~mol} \mathrm{~L}^{-1}$} \\
\hline $0,0-0,05$ & $3,9 \mathrm{bA}$ & $4,1 \mathrm{bA}$ & $4,1 \mathrm{bcA}$ & $4,0 \mathrm{aB}$ & $4,0 \mathrm{cB}$ & $4,5 \mathrm{aA}$ & 3 \\
\hline $0,05-0,1$ & $4,0 \mathrm{bA}$ & $4,2 \mathrm{abA}$ & $4,1 \mathrm{cA}$ & $3,9 \mathrm{aB}$ & $4,0 \mathrm{cB}$ & $4,4 \mathrm{abA}$ & 3 \\
\hline $0,1-0,2$ & $4,1 \mathrm{aA}$ & $4,2 \mathrm{abA}$ & $4,2 \mathrm{abcA}$ & $3,9 \mathrm{aB}$ & $4,1 \mathrm{bcA}$ & $4,2 \mathrm{cA}$ & 1 \\
\hline $0,2-0,3$ & $4,1 \mathrm{aB}$ & $4,2 \mathrm{abAB}$ & $4,4 \mathrm{abA}$ & $4,0 \mathrm{aB}$ & $4,2 \mathrm{abAB}$ & $4,3 \mathrm{bcA}$ & 2 \\
\hline $0,3-0,4$ & $4,1 \mathrm{aB}$ & $4,3 \mathrm{aAB}$ & $4,5 \mathrm{aA}$ & $4,1 \mathrm{aB}$ & $4,3 \mathrm{aA}$ & $4,3 \mathrm{bcA}$ & 1 \\
\hline $\mathrm{CV}(\%)$ & 1 & 1 & 2 & 3 & 2 & 2 & \\
\hline
\end{tabular}

${ }^{(1)}$ Médias seguidas de letras iguais, dentro de cada sistema de colheita, minúsculas nas colunas e maiúsculas nas linhas, não diferem entre si pelo teste de Tukey, a $5 \%$ de probabilidade.

Tabela 3. Capacidade de troca de cátions e saturação por bases em Latossolo Vermelho distrófico sob colheita de cana-deaçúcar com e sem queima ${ }^{(1)}$.

\begin{tabular}{|c|c|c|c|c|c|c|c|}
\hline \multirow{2}{*}{$\begin{array}{l}\text { Camadas } \\
(\mathrm{m}) \\
\end{array}$} & \multicolumn{3}{|c|}{ Com queima } & \multicolumn{3}{|c|}{ Sem queima } & \multirow{2}{*}{$\begin{array}{l}\mathrm{CV} \\
(\%) \\
\end{array}$} \\
\hline & Linha & $0,25 \mathrm{~m}$ & Entrelinha & Linha & $0,25 \mathrm{~m}$ & Entrelinha & \\
\hline \multicolumn{8}{|c|}{ Capacidade de troca de cátions $\left(\mathrm{mmol}_{\mathrm{c}} \mathrm{kg}^{-1}\right)$} \\
\hline $0,0-0,05$ & $83,3 \mathrm{aA}$ & $80,2 \mathrm{abAB}$ & $77,8 \mathrm{aB}$ & $118,8 \mathrm{aA}$ & $109,1 \mathrm{aAB}$ & $104,5 \mathrm{aB}$ & 7 \\
\hline $0,05-0,1$ & $82,0 \mathrm{abA}$ & $84,6 \mathrm{aA}$ & $77,5 \mathrm{aA}$ & $104,5 \mathrm{bA}$ & $101,5 \mathrm{aA}$ & $89,3 \mathrm{bA}$ & 18 \\
\hline $0,1-0,2$ & $77,8 \mathrm{bcA}$ & $74,5 \mathrm{bcA}$ & $72,6 \mathrm{aA}$ & $89,8 \mathrm{cA}$ & $84,9 \mathrm{bA}$ & $82,1 \mathrm{bcA}$ & 9 \\
\hline $0,2-0,3$ & $76,2 \mathrm{cdA}$ & $73,9 \mathrm{bcA}$ & $72,6 \mathrm{aA}$ & $90,7 \mathrm{cA}$ & $87,3 \mathrm{bA}$ & $83,6 \mathrm{bcA}$ & 8 \\
\hline$\underline{0,3-0,4}$ & $72,3 \mathrm{dA}$ & $69,1 \mathrm{cAB}$ & $65,6 \mathrm{bB}$ & $82,3 \mathrm{dA}$ & $82,5 \mathrm{bA}$ & $77,4 \mathrm{cA}$ & 9 \\
\hline CV $(\%)$ & 7 & 12 & 9 & 9 & 10 & 13 & \\
\hline \multicolumn{8}{|c|}{ Saturação por bases (\%) } \\
\hline $0,0-0,05$ & $17,8 \mathrm{bB}$ & $20,6 \mathrm{bB}$ & $24,8 \mathrm{aA}$ & $32,8 \mathrm{aAB}$ & $30,7 \mathrm{abB}$ & $42,4 \mathrm{aA}$ & 5 \\
\hline $0,05-0,1$ & $19,8 \mathrm{bB}$ & $29,7 \mathrm{aA}$ & $22,4 \mathrm{aAB}$ & $28,9 \mathrm{abA}$ & $29,7 \mathrm{abA}$ & $36,0 \mathrm{abA}$ & 6 \\
\hline $0,1-0,2$ & $22,9 \mathrm{aA}$ & $21,8 \mathrm{bA}$ & $24,1 \mathrm{aA}$ & $25,7 \mathrm{abA}$ & $26,0 \mathrm{bA}$ & $28,0 \mathrm{bA}$ & 4 \\
\hline $0,2-0,3$ & $22,7 \mathrm{aA}$ & $23,9 \mathrm{abA}$ & $25,9 \mathrm{aA}$ & $27,4 \mathrm{abA}$ & $29,2 \mathrm{abA}$ & $28,3 \mathrm{bA}$ & 3 \\
\hline $0,3-0,4$ & $22,0 \mathrm{aB}$ & $26,6 \mathrm{abA}$ & $28,7 \mathrm{aA}$ & $25,3 \mathrm{bB}$ & $31,4 \mathrm{aA}$ & $30,5 \mathrm{bA}$ & 2 \\
\hline$\overline{C V}(\%)$ & 3 & 5 & 5 & 4 & 3 & 5 & \\
\hline
\end{tabular}


mascarou possíveis diferenças localizadas nas camadas de $0-0,05$ e de $0,05-0,1 \mathrm{~m}$. Esses resultados também podem ser explicados pela elevada movimentação dos cátions no perfil do Argissolo e avaliado por Mendonza et al. (2000), já que esse solo era mais arenoso do que o utilizado no presente trabalho.

Valores de $\mathrm{V}$ das amostras de solo não diferiram entre as posições de coleta, nas três camadas intermediárias, para ambos os sistemas. Na camada de $0-0,05 \mathrm{~m}$, o solo coletado na entrelinha apresentou $\mathrm{V}$ superior ao encontrado nas amostras dos locais linha e a $0,25 \mathrm{~m}$ da linha (Tabela 3), possivelmente um reflexo da maior absorção de cátions básicos do complexo sortivo pelas plantas, nos dois últimos locais. Na camada mais profunda $(0,3-0,4 \mathrm{~m})$, os valores foram mais elevados a $0,25 \mathrm{~m}$ da linha e na entrelinha, em decorrência da menor acidificação nesses locais do que na linha de plantio (Chien et al., 2008).

Os valores de P-disponível extraídos pela resina nas amostras dos tratamentos SQ e CQ não diferiram na maioria das posições de coleta, nas camadas avaliadas (Figura 3). A exceção foi na camada de 0-0,05 $\mathrm{m}$ da posição $0,25 \mathrm{~m}$, em que o P-resina na área CQ foi mais elevado, possivelmente em razão da rápida disponibilização do $\mathrm{P}$ pela queima da palha, conforme observado por Ball-Coelho et al. (1993).
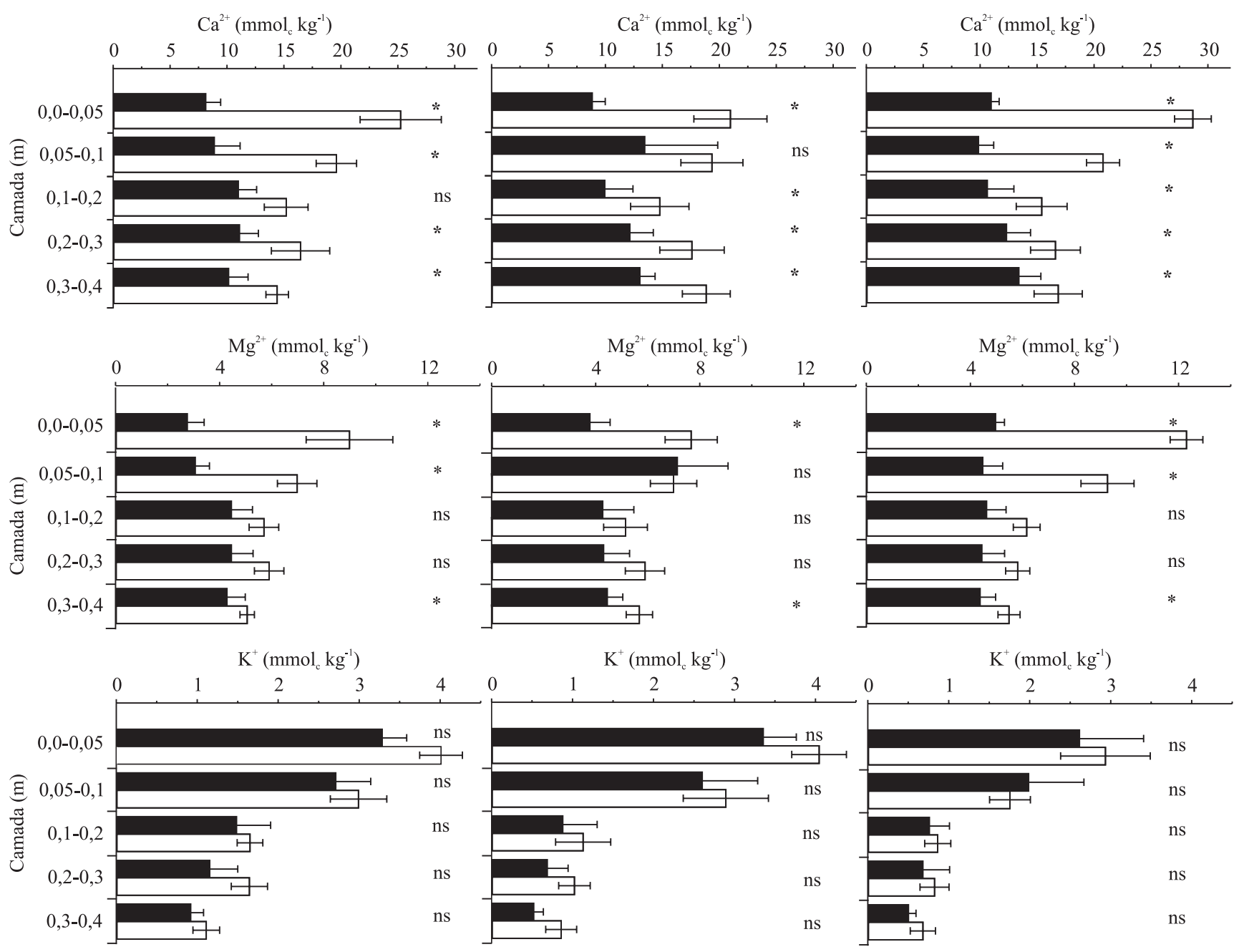

Linha

$0,25 \mathrm{~m}$

Entrelinha

Com queima $\longleftarrow$ Sem queima

Figura 2. Teores de $\mathrm{Ca}^{2+}, \mathrm{Mg}^{2+}$ e $\mathrm{K}^{+}$em Latossolo Vermelho distrófico sob colheita de cana-de-açúcar com e sem queima. ${ }^{n s}$ Não significativo. *Diferenças significativas entre os sistemas com e sem queima pelo teste de Tukey, a 5\% de probabilidade. As barras representam o erro-padrão da média. 
Czycza (2009) também não observou diferenças nos teores de P na camada de 0-0,1 m, entre os dois tratamentos CQ e SQ, após 6 e 12 anos. Nas camadas de $0,1-0,2$ e $0,2-0,3 \mathrm{~m}$, o autor encontrou quantidades de P-resina superiores nas áreas sob cultivo SQ, em comparação ao sistema CQ. Os teores de P-resina não diferiram entre locais de coleta em ambos os sistemas, na camada de $0-0,3 \mathrm{~m}$ (Tabela 4). Na camada subsequente, de 0,3-0,4 m, verificou-se tendência de maior concentração do elemento na linha de plantio, o que indica o efeito residual da adubação de plantio no fundo do sulco em ambos os sistemas de colheita. Com relação às profundidades de amostragem, houve maior concentração nas camadas de $0,2-0,3$ e $0,3-0,4 \mathrm{~m}$ na posição linha, enquanto nas posições $0,25 \mathrm{~m}$ e entrelinha, não houve diferença entre as cinco camadas avaliadas.

O solo sob cana-de-açúcar CQ apresentou maiores quantidades de P-disponível extraído pela solução Mehlich-1 na camada superficial $(0-0,05 \mathrm{~m})$, nas três posições de coleta, e na camada de $0,05-0,1 \mathrm{~m}$, na linha de plantio (Figura 3). Os maiores valores nas amostras de solo sob sistema CQ podem ser explicados pelo fosfato de cálcio formado durante a queima da palha e presente nas cinzas depositadas no solo (Ball-Coelho et al., 1993).

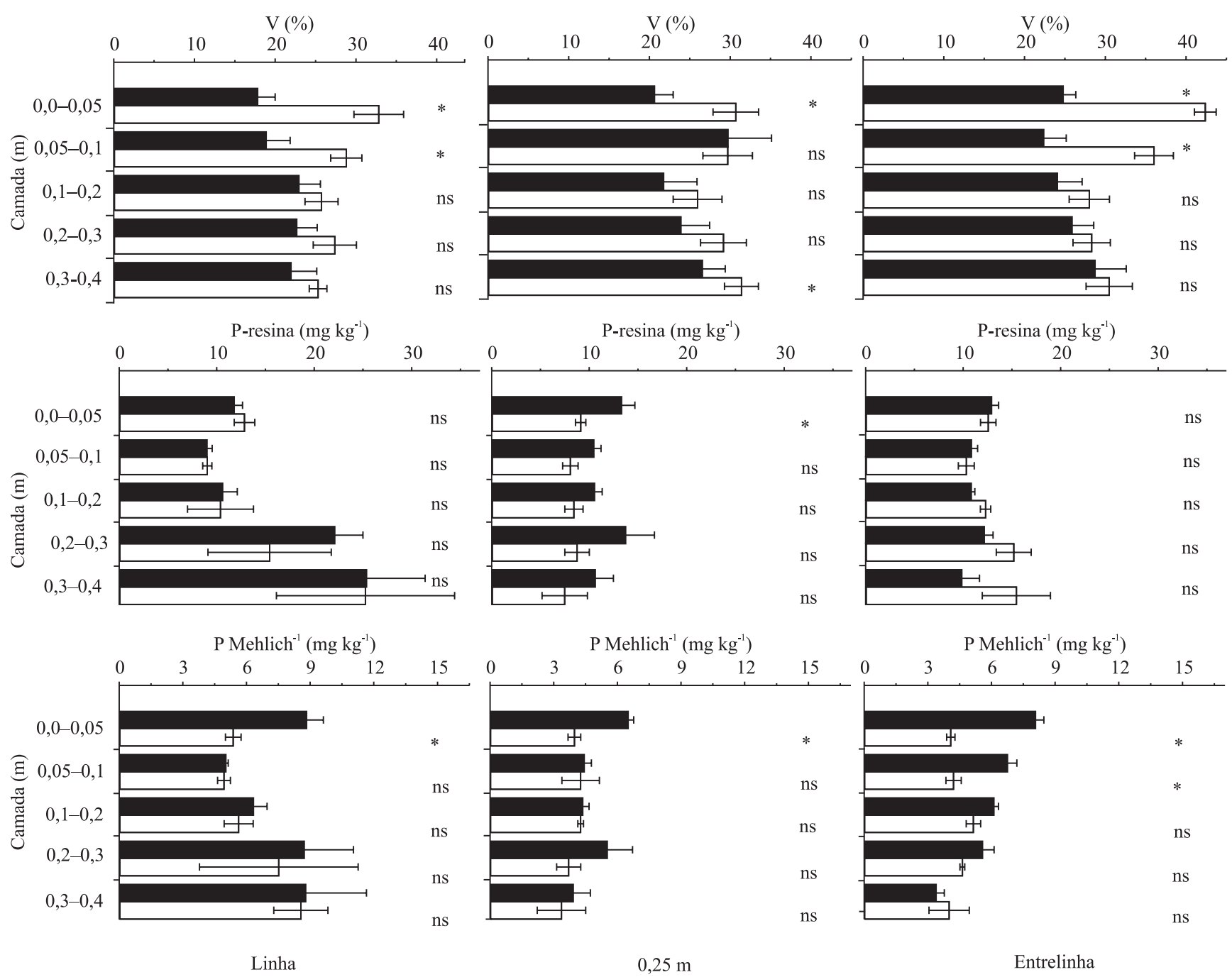

Com queima $\longleftarrow$ Sem queima

Figura 3. Saturação por bases (V) e P-disponível pelo extrator resina e Mehlich 1 em um Latossolo Vermelho distrófico

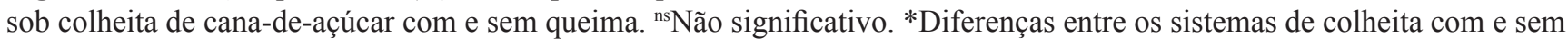
queima pelo teste de Tukey a $5 \%$ de probabilidade. As barras representam o erro-padrão da média. 
Tabela 4. Teores de P-disponível em um Latossolo Vermelho distrófico sob colheita de cana-de-açúcar com e sem queima $^{(1)}$.

\begin{tabular}{|c|c|c|c|c|c|c|c|}
\hline \multirow{2}{*}{$\begin{array}{l}\text { Camadas } \\
\text { (m) }\end{array}$} & \multicolumn{3}{|c|}{ Com queima } & \multicolumn{3}{|c|}{ Sem queima } & \multirow{2}{*}{$\begin{array}{l}\mathrm{CV} \\
(\%)\end{array}$} \\
\hline & Linha & $0,25 \mathrm{~m}$ & Entrelinha & Linha & $0,25 \mathrm{~m}$ & Entrelinha & \\
\hline \multicolumn{8}{|c|}{ P-resina $\left(\mathrm{mg} \mathrm{kg}^{-1}\right)$} \\
\hline $0,0-0,05$ & $11,8 \mathrm{bA}$ & $13,3 \mathrm{aA}$ & $12,9 \mathrm{aA}$ & $12,9 \mathrm{bcA}$ & $9,1 \mathrm{aB}$ & $12,6 \mathrm{aA}$ & 7 \\
\hline $0,05-0,1$ & $9,0 \mathrm{bA}$ & $10,5 \mathrm{aA}$ & $10,9 \mathrm{aA}$ & $9,0 \mathrm{cA}$ & $8,1 \mathrm{aA}$ & $10,3 \mathrm{aA}$ & 10 \\
\hline $0,1-0,2$ & $10,6 \mathrm{bA}$ & $10,6 \mathrm{aA}$ & $10,8 \mathrm{aA}$ & $10,4 \mathrm{bcA}$ & $8,4 \mathrm{aA}$ & $12,3 \mathrm{aA}$ & 14 \\
\hline $0,2-0,3$ & $22,1 \mathrm{aA}$ & $13,8 \mathrm{aA}$ & $12,2 \mathrm{aA}$ & $15,4 \mathrm{abA}$ & $8,8 \mathrm{aA}$ & $15,2 \mathrm{aA}$ & 18 \\
\hline $0,3-0,4$ & $25,4 \mathrm{aA}$ & $10,6 \mathrm{aAB}$ & $9,9 \mathrm{aB}$ & $25,3 \mathrm{aA}$ & $7,5 \mathrm{aB}$ & $15,5 \mathrm{aAB}$ & 23 \\
\hline CV (\%) & 13 & 13 & 10 & 15 & 12 & 13 & \\
\hline \multicolumn{8}{|c|}{ P Mehlich-1 $\left(\mathrm{mg} \mathrm{kg}^{-1}\right)$} \\
\hline $0,0-0,05$ & $8,8 \mathrm{aA}$ & $6,5 \mathrm{aB}$ & $8,1 \mathrm{aAB}$ & $5,4 \mathrm{abA}$ & $4,0 \mathrm{aB}$ & $4,1 \mathrm{aB}$ & 6 \\
\hline $0,05-0,1$ & $5,0 \mathrm{aAB}$ & $4,4 \mathrm{abB}$ & $6,8 \mathrm{abA}$ & $4,9 \mathrm{bA}$ & $4,3 \mathrm{aA}$ & $4,2 \mathrm{aA}$ & 10 \\
\hline $0,1-0,2$ & $6,3 \mathrm{aA}$ & $4,4 \mathrm{abB}$ & $6,1 \mathrm{abA}$ & $5,6 \mathrm{abA}$ & $3,8 \mathrm{aB}$ & $5,1 \mathrm{aA}$ & 7 \\
\hline $0,2-0,3$ & $8,7 \mathrm{aA}$ & $5,5 \mathrm{abA}$ & $5,6 \mathrm{bA}$ & $7,5 \mathrm{abA}$ & $3,7 \mathrm{aB}$ & $4,6 \mathrm{aAB}$ & 20 \\
\hline $0,3-0,4$ & $8,8 \mathrm{aA}$ & $3,9 \mathrm{bA}$ & $3,4 \mathrm{cA}$ & $8,6 \mathrm{aA}$ & $3,4 \mathrm{aA}$ & $4,0 \mathrm{aA}$ & 27 \\
\hline$\overline{\mathrm{CV}}(\%)$ & 18 & 13 & 8 & 15 & 18 & 13 & \\
\hline
\end{tabular}

${ }^{(1)}$ Médias seguidas de letras iguais, dentro de cada sistema de colheita, minúsculas nas colunas e maiúsculas nas linhas, não diferem entre si pelo teste de Tukey, a $5 \%$ de probabilidade

Mendonza et al. (2000) determinaram teor de P-Mehlich 2,5 vezes maior na área SQ, na camada de $0-0,1 \mathrm{~m}$, e valores iguais ao tratamento CQ até $0,6 \mathrm{~m}$ de profundidade, ao avaliar um Argissolo arenoso, com $\mathrm{pH}$ em torno de 6,0, cultivado por cinco anos com cana-de-açúcar SQ. Esses resultados podem ser atribuídos às diferenças nas condições experimentais, como período de utilização do manejo de colheita sem queima, nas condições de fertilidade e nas classes texturais dos solos.

\section{Conclusões}

1. Os valores de carbono orgânico total, capacidade de troca catiônica, teores de $\mathrm{Ca}^{2+}$ e $\mathrm{Mg}^{2+}$ e saturação por bases são mais elevados no sistema de colheita da cana-de-açúcar sem queima da palha do que no com queima, especialmente nas camadas mais superficiais.

2. Os valores dos atributos de fertilidade do solo apresentam maiores diferenças entre os locais de amostragem na camada superficial, de $0-0,05 \mathrm{~m}$ de profundidade.

3. A colheita sem queima favorece o aumento da fertilidade do solo, exceto quanto aos teores de $\mathrm{P}$ disponível.

\section{Agradecimentos}

À Coordenação de Aperfeiçoamento de Pessoal de Nível Superior e ao Conselho Nacional de
Desenvolvimento Científico e Tecnológico, pelo apoio financeiro; e ao Programa de Pós-graduação em Solos e Nutrição de Plantas da Escola Superior de Agricultura Luiz de Queiroz, pelo apoio durante a pesquisa.

\section{Referências}

ALVAREZ, I.A.; CASTRO, P.R. de C. e; NOGUEIRA, M.C.S. Crescimento de raízes de cana crua e queimada em dois ciclos. Scientia Agricola, v.57, p.653-659, 2000.

ANGHINONI, I. Fertilidade do Solo e seu manejo no sistema plantio direto. In: NOVAIS, R.F.; ALVAREZ V., V.H.; BARROS, N.F. de; FONTES, R.L.F.; CANTARUTTI, R.B.; NEVES, J.C.L. (Ed.). Fertilidade do solo. Viçosa: Sociedade Brasileira de Ciência do Solo, 2007. p.873-928.

BALL-COELHO, B.; SALCEDO, I.H.; TIESSEN, H.; STEWART, J.W.B. Short- and long-term phosphorus dynamics in a fertilized Ultisol under sugarcane. Soil Science Society of America Journal, v.57, p.1.027-1.034, 1993.

BAYER, C.; MIELNICZUK, J. Dinâmica e função da matéria orgânica. In: SANTOS, G. de A.; SILVA, L.S. da; CANELLAS, L.P.; CAMARGO, F. de O. (Ed.). Fundamentos da matéria orgânica do solo: ecossistemas tropicais e subtropicais. 2.ed. Porto Alegre: Metrópole, 2008. p.7-18.

CANELLAS, L.P.; BUSATO, J.G.; DOBBSS, L.B.; BALDOTTO, A.; RUMJANEK, V.M.; OLIVARES, F.L. Soil organic matter and nutrient pools under long-term non-burning management of sugar cane. European Journal of Soil Science, v.61, p.375-233, 2010.

CHIEN, S.H.; GEARHART, M.M.; COLLAMER, D.J. The effect of different ammonical nitrogen sources on soil acidification. Soil Science, v.173, p.544-551, 2008. 
COLONEGO, J.C.; FOLONI, J.S.S.; ROSOLEM, C.A. Lixiviação de potássio da palha de plantas de cobertura em diferentes estádios de senescência após a dessecação química. Revista Brasileira de Ciência do Solo, v.29, p.99-108, 2005.

CZYCZA, R.V. Quantidade e qualidade da matéria orgânica do solo em sistemas de colheita com e sem queima da cana-de-açúcar. 2009. 92p. Dissertação (Mestrado) - Escola Superior de Agricultura Luiz de Queiroz, Piracicaba.

LUCA, E.F. de; FELLER, C.; CERRI, C.C.; BARTHÈS, B.; CHAPLOT, V.; CAMPOS, D.C.; MANECHINI, C. Avaliação de atributos físicos e estoques de carbono e nitrogênio em solos com queima e sem queima do canavial. Revista Brasileira de Ciência do Solo, v.32, p.789-800, 2008.

MALAVOLTA, E. ABC da análise de solo e folhas: amostragem, interpretação e sugestões de adubação. São Paulo: Agronômica Ceres, 1992. 124p.

MANUAL de adubação e de calagem para os Estados do Rio Grande do Sul e de Santa Catarina. 10.ed. Porto Alegre: Sociedade Brasileira de Ciência do Solo, 2004. 400p.

MENDONZA, H.N.S.; LIMA, E.; ANJOS, L.H.C.; SILVA, L.A.; CEDDIA, M.B., ANTUNES, M.V.M. Propriedades químicas e biológicas de solo de tabuleiro cultivado com cana-de-açúcar com e sem queima da palhada. Revista Brasileira de Ciência do Solo, v.24, p.201-207, 2000.

OLIVEIRA, M.W. de; TRIVELIN, P.C.O.; PENATTI, C.P.; PICCOLO, M. de C. Decomposição e liberação de nutrientes da palhada de cana-de-açúcar em campo. Pesquisa Agropecuária Brasileira, v.34, p.2359-2362, 1999.

RAIJ, B. van; CANTARELLA, H.; QUAGGIO, J.A.; FURLANI, A.M.C. (Ed.). Recomendações de adubação e calagem para o estado de São Paulo. 2.ed. Campinas: Instituto Agronômico, 1997. 285p. (IAC. Boletim técnico, 100).
RAZAFIMBELO, T.; BARTHÈS, B.; LARRÉ-LARROUY, M.C.; LUCA, E.F.; LAURENT, J.Y.; CERRI, C.C.; FELLER, C. Effect of sugarcane residue management (mulching versus burning) on organic matter in a clayey Oxisol from southern Brazil. Agriculture Ecosystems and Environment, v.115, p.285-289, 2006.

ROSOLEM, C.A.; CALONEGO, J.C. ; FOLONI, J.S.S.; GARCIA, R.A. Potássio lixiviado da palha de aveia-preta e milheto após a dessecação química. Pesquisa Agropecuária Brasileira, v.42, p.1169-1175, 2007.

SANTOS, H.G. dos; JACOMINE, P.K.T.; ANJOS, L.H.C. dos; OLIVEIRA, V.A. de; OLIVEIRA, J.B. de; COELHO, M.R.; LUMBRERAS, J.F.; CUNHA, T.J.F. (Ed.). Sistema brasileiro de classificação de solos. 2.ed. Rio de Janeiro: Embrapa Solos, 2006. $306 \mathrm{p}$.

SAS INSTITUTE. SAS/STAT software: changes and enhancements through release 6.12. Version 9.2. Cary: SAS Institute, 1997. $1167 \mathrm{p}$.

SILVA, I.R.; MENDONÇA, E.S. Matéria orgânica do solo. In.: NOVAIS, R.F.; ALVAREZ V., V.H.; BARROS, N.F. de; FONTES, R.L.F.; CANTARUTTI, R.B.; NEVES, J.C.L (Ed.). Fertilidade do solo. Viçosa: Sociedade Brasileira de Ciência do Solo, 2007. p.275-374.

VITTI, G.C.; MAZZA, J.A. Planejamento, estratégias de manejo e nutrição da cana-de-açúcar. Piracicaba: Potafos, 2002. 16p. (Encarte técnico/Informações agronômicas, 97).

WOOD, A.W. Management of crop residues following green harvesting of sugarcane in north Queensland. Soil and Tillage Research, v.20, p.69-85, 1991.

ZINN, Y.L.; LAL, R.; RESCK, D.V.S. Changes in soil organic carbon stocks under agriculture in Brazil. Soil and Tillage Research, v.84, p.25-40, 2005. 